\title{
The role of the optical coherence tomography in identifying shape and size of idiopathic epiretinal membranes
}

\author{
Bilal Hajnajeeb, Michael Georgopoulos, Ramzi Sayegh, Wolfgang Geitzenauer, \\ Ursula Schmidt-Erfurth
}

Department of Ophthalmology Medical University of Vienna, Vienna, Austria

\section{Correspondence to Professor Dr Michael Georgopoulos, Department of Ophthalmology, Medical University of Vienna, Spitalgasse 23, Vienna A-1090, Austria; michael. georgopoulos@meduniwien.ac. at}

Accepted 28 December 2011 Published Online First 10 February 2012

\begin{abstract}
AIM Currently, the border of idiopathic epiretinal membranes (iERM) is outlined intraoperatively using vital dyes. Therefore, the authors set out to investigate the role of the preoperative retinal thickness map (RTM) of the optical coherence tomography (OCT) in identifying the shape and the size of the iERMs.

Methods 15 eyes of 15 patients with IERM who underwent vitrectomy with indocyanine green-assisted membrane peeling were included in this study. The authors analysed the intraoperative fundus images and preoperative Cirrus HD-OCT to detect the shape and the size of the iERM as well as the shape and the size of each thickness-indicating colour (white, red, orange and yellow) on the RTM, respectively. The correlation of areas and morphologic characteristics between both groups was explored.
\end{abstract}

Results Analysis of iERM morphologic characteristics (shape) showed a similarity between the iERM contour and the corresponding RTM in 13 cases (86.6\%).

Furthermore, retinal folds were found in six iERMs and in their corresponding RTMs. Analysis of iERM size (area) revealed a positive correlation between the iERM area and each studied coloured area in RTM. The most significant correlation was between iERM and the red area $(440-480 \mu \mathrm{m} ; \mathrm{r}=0.87, \mathrm{p}<0.0001)$.

Conclusion The iERM-related retinal folds are clearly distinguishable on the HD-OCT. The red area in RTM representing the $440-480 \mu \mathrm{m}$ retinal thickness can be a reliable predictor of the extent and the shape of the iERM.

\section{INTRODUCTION}

Since the first demonstrations of Huang et al in $1991,{ }^{1}$ the optical coherence tomography (OCT) technology has changed our understanding and consequently our practice of ophthalmology. ${ }^{2}$ Recently, a significant part of published OCT studies concerned the management of idiopathic epiretinal membrane (iERM) and vitreomacular interface (VMI) diseases. The high sensitivity of $\mathrm{OCT}^{3}$ as well as its very detailed three dimensional imaging when compared with stereoscopic ophthalmoscopy or photography ${ }^{4}$ allows clinicians to use this unique, non-invasive technique to fully visualise the dynamics of the epiretinal traction and the subtle structures in and around the VMI. ${ }^{67}$ As a result, the applications of the modern versions of the OCT have been extended beyond the diagnostic decision-making stage to more sophisticated purposes. Many studies have clearly confirmed the pivotal role of the OCT in all three main steps in the epiretinal membrane (ERM) management: (1) preoperatively: giving a comprehensive view of the pathophysiology of the ERM ${ }^{3}$ and additionally to provide a fine structural assessment of the retinal layers and the $\mathrm{VMI}^{8}{ }^{9}$ (2) intraoperatively: guidance of the surgeon, based on preoperative OCT images, in the membrane peeling process; $;^{11}$ and (3) postoperatively: predicting the course of macula recovery after membrane peeling including the prognosis and the expected final visual outcome. ${ }^{8} 10^{12-14}$

Currently, the most reliable way to outline the border of the (semi)transparent ERM can be done only intraoperatively by vital dyes. These dyes are commonly used as adjuncts to aid in the removal of the ERM and/or the internal limiting membrane (ILM) during the membrane peeling surgery. ${ }^{15} 16$ Indocyanine green (ICG) is a commonly used intravitreal chromophore that delineates ERMs through its affinity to the ILM exposed to the dye at the margins of the epiretinal contact.

Given the increasing dependence on OCT in the management of iERMs and the challenge to provide a safe and reliable preoperative evaluation of the shape and size of the ERM, we aimed to investigate the role of the preoperative retinal thickness map (RTM) of the OCT in identifying the shape and the size of the iERM.

\section{METHODS}

This retrospective study included 27 eyes of 27 consecutive iERM patients who underwent vitrectomy with ICG-assisted membrane peeling with intraoperative photo documentation (Olympus, Visera, OTV-S7, Tokyo, Japan) in the Department of Ophthalmology of the Medical University of Vienna. All the research and measurements were performed in accordance with the tenets of the Declaration of Helsinki. The ERM diagnosis was made following standard methods including slit lamp fundus biomicroscopy, fundus photography and OCT examination with Cirrus HD-OCT (Carl Zeiss Meditec, Dublin, California, USA).

Inclusion criteria: patients demonstrating distinct ICG staining with good quality intraoperative photos, no considerable elevation of the iERM from the underlying surface of the retina and HD-OCT images taken within 30 days preoperatively without any retinal thickness measurement errors were included.

Exclusion criteria: patients who had at least one of the following: patchy ICG staining, bad quality 
intraoperative photos, considerable elevation of the iERM from the underlying surface of the retina, HD-OCT images older than 30 days preoperatively or retinal thickness measurement errors.

Of all studied eyes, the operative decision was based on disturbing metamorphopsia, diplopia or reduced visual acuity. The level of the increase in thickness of the maculae was not a concern.

Histologically, ICG outlines the ERM by staining selectively the exposed ILM beyond the iERM margin. ${ }^{17}$ Thus, these photos illustrated a central unstained iERM surface surrounded by a green dyed iERM-free neighbouring retina (figure $1 \mathrm{~A}, \mathrm{~B}$ ). To make these photos evaluable and comparable with the different thickness macular measurements obtained by RTM, each photo had to be edited (well rotated and resized) to fit exactly its corresponding enface OCT image, which in turn represents the definitive position and size of the corresponding RTM (figure $1 \mathrm{C}, \mathrm{D})$. Therefore, the investigators were blinded to neither the OCT images nor the intraoperative photos.

We identified the iERM area on intraoperative images as the unstained central area outlined by ICG-stained retina. Similarly, each colour on OCT-RTM (white, red, orange and yellow coded areas), representing an area with a specific range of retinal thicknesses, was compared with the iERM contour.

\section{Analysis of iERM shape}

Subjectively, we assessed the shape similarity between each unstained central area on the intraoperative images and its corresponding thickness-indicating colours map. We evaluated it as yes if the whole unstained IERM area including its contour looks similar to its corresponding RTM, and no if there was no obvious similarity. In addition, the presence and direction of any retinal folds were also determined by two certified ophthalmologists.

\section{Analysis of iERM size}

The unstained macular surface (iERM) areas of the edited intraoperative photos as well as each pathological false colour coding (white, red, orange, yellow) on the $(6 \times 6 \mathrm{~mm})$ RTM were measured with the assistance of Image J program (freeware, National Institutes of Health, Bethesda, Maryland, USA). Subsequently, we used a predefined excel program that converts the pixel into quadratic micrometre size. The correlation between both groups and the mean of all calculated areas were explored. The correlation between iERM and thickness-indicating colours was determined by the Pearson correlation test. The reported $p$ value is a result of a two-sided test. A $p$ value of $\mathrm{p} \leq 0.05$ was considered significant.

\section{RESULTS}

According to the exclusion criteria, 12 of 27 patients were excluded from the study: five patients had patchy ICG staining on the intraoperative photos, three patients had bad quality intraoperative photos, two patients had OCT images older than 30 days, one patient had RTM error on the OCT image and one patient had RTM error on the OCT image as well as considerable elevation of the iERM from the underlying retina. Fifteen eyes of the remaining 15 patients (seven women, eight men) were therefore included in our survey.

Analysis of the iERM morphologic characteristics (configuration): of all 15 examined subjects, we noticed a similarity between iERM shape and its corresponding RTM in 13 cases (86.6\%). In addition, we detected retinal folds in six intraoperative photos and in their corresponding RTMs. The directions of the folds were radial to the nasal side in four cases (figure 1D), vertical in one case and star-like in one case.

Analysis of the ERM size (area): the iERM area was positively and significantly correlated with yellow $(360-400 \mu \mathrm{m})$ and orange $(400-440 \mu \mathrm{m})$ retinal thickness-colour coding on RTM (r: $0.68, \mathrm{r}: 0.83 ; \mathrm{p}<0.05)$ respectively, and most significantly correlated with the red $(440-480 \mu \mathrm{m})$ retinal thickness coding ( $\mathrm{r}$ : 0.87; $\mathrm{p}$ 0.0001) (figure 2). Whereas, the modest correlation ( $\mathrm{r}$ : $0.41)$ with the white thickness area $(>480 \mu \mathrm{m})$ was statistically
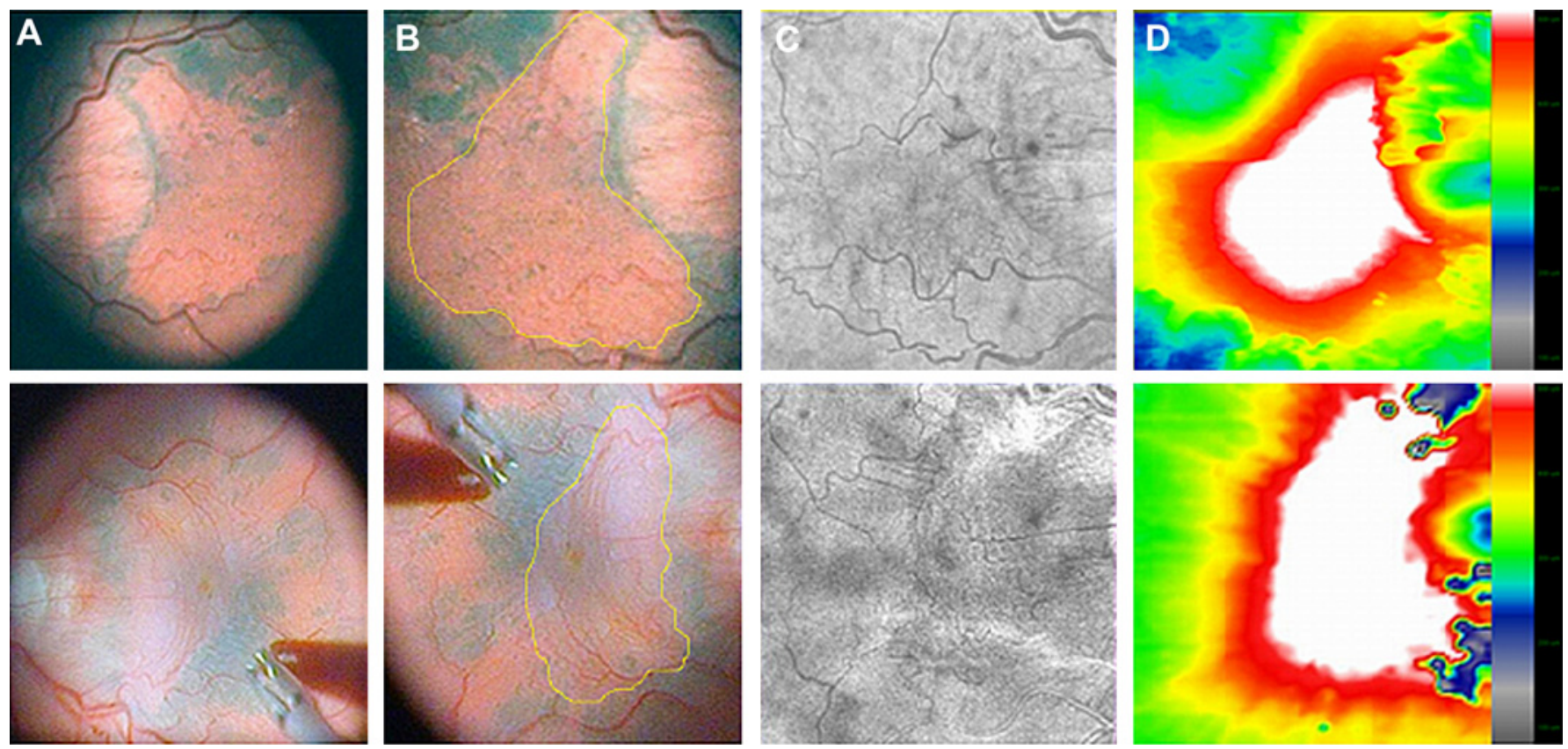

Figure 1 Illustrates the editing steps of the images of two patients. A, The intraoperative photos before editing. B, The photos after being rotated and resized according to their en face image. Note the central unstained epiretinal membrane (ERM) surface surrounded by a green ERM-free margin. $C$, The corresponding en face images of the macula taken 1 day before surgery. D, Retinal thickness map of its corresponding intraoperative image in $B$, with retinal folds directing to the nasal side. 
Figure 2 The scatter plot diagrams of the four studied correlations with correlation coefficient $r$ and $p$ values. Note the progressive increase of the correlation coefficient during the gradual increase of the macular thickness from yellow to red thicknesscolour coding. ERM, epiretinal membrane.
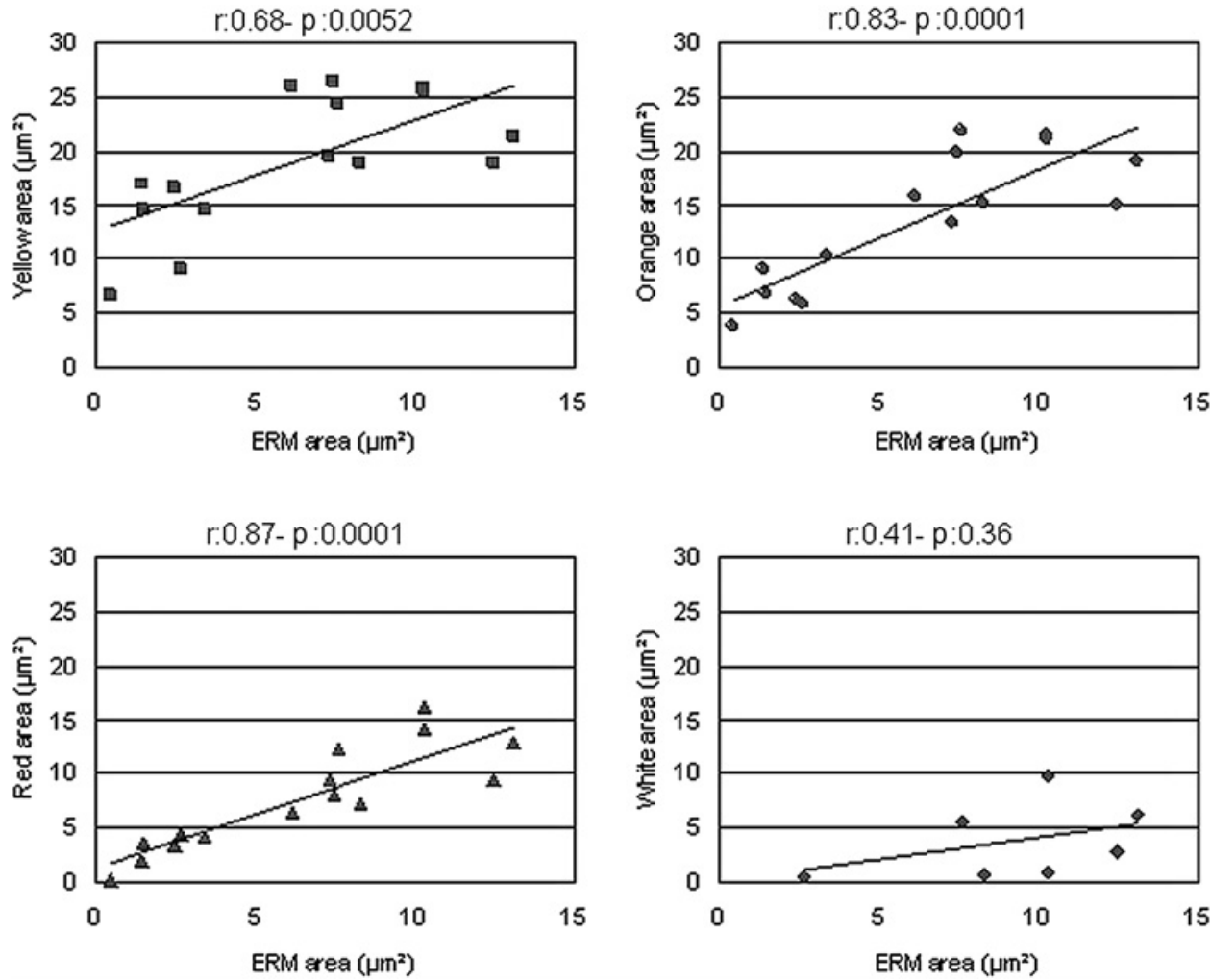

insignificant, as only seven of 15 examined eyes had a retinal thickness of more than $480 \mu \mathrm{m}$ (table 1).

On RTM, the means of the thickness-indicating coloured (yellow, orange, red and white) areas were 19.01, 13.57, 7.58 and $3.87 \mu \mathrm{m}^{2}$ respectively, while the mean intraoperative iERM area was $6.32 \mu \mathrm{m}^{2}$ (table 1).

\section{DISCUSSION}

Regarding the shape of the membrane, RTM revealed a high ability to determine retinal folds and their direction seen intraoperatively and manifested a high proportion of similarity $(86.6 \%)$ with the iERM as delineated intraoperatively with ICG.
RTM could provide useful information concerning the presumed shape of iERM preoperatively.

In respect of the size of the $\mathrm{ERRM}$, we found that an increase in the pathological retinal thickness from 360 to $480 \mu \mathrm{m}$ is accompanied by a proportional increase in the correlation with the iERM area. Interestingly, the correlation was most significant ( $\mathrm{r}: 0.87 ; \mathrm{p}$ 0.0001) at a retinal thickness range of $440-480 \mu \mathrm{m}$ (the red area). When compared with the means of other areas on RTM, the red coded area $\left(7.58 \mu \mathrm{m}^{2}\right)$ in preoperative images was the closest area to the intraoperative ERM area $\left(6.32 \mu \mathrm{m}^{2}\right)$. The high correlations between the ERM (the cause) and RTM size (the consequence) can help to realistically predict

Table 1 Results of the size and shape analysis of both the intraoperative aspect of the epiretinal membrane (ERM) and the preoperative white, red, orange and yellow thickness-colour coding in the retinal thickness map (RTM)

\begin{tabular}{|c|c|c|c|c|c|c|c|c|}
\hline Patient No. & $\begin{array}{l}\text { ERM areas } \\
\left(\mu \mathrm{m}^{2}\right)\end{array}$ & $\begin{array}{l}\text { White areas } \\
\left(\mu \mathrm{m}^{2}\right)\end{array}$ & $\begin{array}{l}\text { Red areas } \\
\left(\mu \mathrm{m}^{2}\right)\end{array}$ & $\begin{array}{l}\text { Orange areas } \\
\left(\mu \mathrm{m}^{2}\right)\end{array}$ & $\begin{array}{l}\text { Yellow areas } \\
\left(\mu \mathrm{m}^{2}\right)\end{array}$ & $\begin{array}{l}\text { Direction of folds on } \\
\text { microscopic photo }\end{array}$ & $\begin{array}{l}\text { Retinal folds } \\
\text { on RTM }\end{array}$ & $\begin{array}{l}\text { ERM/RTM } \\
\text { similarity }\end{array}$ \\
\hline 1 & 1.42 & 0 & 1.92 & 9.15 & 16.99 & Star-like towards fovea & Yes & No \\
\hline 2 & 2.44 & 0 & 3.4 & 6.28 & 16.6 & No folds & No & No \\
\hline 3 & 1.51 & 0 & 3.7 & 6.89 & 14.51 & No folds & No & Yes \\
\hline 4 & 2.68 & 0.61 & 4.5 & 5.76 & 9 & Radial to nasal & Yes & Yes \\
\hline 5 & 3.43 & 0 & 4.15 & 10.25 & 14.58 & Radial to nasal & Yes & Yes \\
\hline 6 & 7.35 & 0 & 9.5 & 13.26 & 19.63 & No folds & No & Yes \\
\hline 7 & 8.3 & 0.88 & 7.22 & 15.09 & 18.97 & No folds & No & Yes \\
\hline 8 & 7.44 & 0 & 8.13 & 19.83 & 26.42 & No folds & No & Yes \\
\hline 9 & 6.18 & 0 & 6.5 & 15.64 & 25.96 & No folds & No & Yes \\
\hline 10 & 12.47 & 2.83 & 9.5 & 14.89 & 18.89 & No folds & No & Yes \\
\hline 11 & 13.1 & 6.26 & 12.92 & 18.89 & 21.26 & Radial to nasal & Yes & Yes \\
\hline 12 & 7.57 & 5.66 & 12.15 & 21.76 & 24.31 & No folds & No & Yes \\
\hline 13 & 10.25 & 1.03 & 13.85 & 21.32 & 25.65 & Radial to nasal & Yes & Yes \\
\hline 14 & 10.3 & 9.8 & 16.08 & 20.93 & 25.76 & Vertical & Yes & Yes \\
\hline 15 & 0.5 & 0 & 0.28 & 3.74 & 6.63 & No folds & No & Yes \\
\hline Mean & 6.32 & 3.87 & 7.58 & 13.57 & 19.01 & & & \\
\hline
\end{tabular}


the size of the ERM using regression biostatistics dependent on RTM red area.

Unlike the secondary ERM which demonstrates focal adhesion points, Mori and associates reported that iERM tends to have a contiguous adhesion to the underlying retina. ${ }^{18}$ Furthermore, Legarreta et al studied the segmented surface maps of ERM and ILM separately to evaluate the tangential tractive effect of the ERM on the retinal architecture. ${ }^{19}$ They revealed that tension lines are more frequent and clustered together where an overlying ERM is present, and that these lines are spread out where ERM is absent. Hence, we can explain the high similarity and the positive correlation noted in our results between intraoperative iERMs and corresponding RTMs as the thickening of the macula, which is seen by RTM, is mainly a consequence of the adherent tangential tractive forces of the iERM exerted on the underlying macula. Subsequently, the thickening of the macula is restricted to the area of the overlying iERM. Nevertheless, the dissimilarity between RTMs and their corresponding iERMs, noticed in the first two patients, can be explained by the involvement of other accompanying traction factors (eg, the posterior vitreous). However, the tractive effect of the posterior vitreous can only be verified by B scan but not by RTM. ${ }^{4} 6$

Nowadays, the method of choice for the treatment of symptomatic ERM is pars plana vitrectomy with membrane peeling using vital dyes. Using vital dyes (ICG and trypan blue) is one of the most important developments in macular hole and ERM surgery. This technique has enabled surgeons to perform ILM and ERM peeling with improved ease and significantly reduced the duration of surgery and the trauma to the nerve fibres layer. ${ }^{20}$ However, adverse effects are still being reported. $^{21} 22$ Many recent reports advocate a long-term postoperative observation of patients to detect any possible functional adverse events. ${ }^{23}$ Therefore, a new trend has recently risen to replace them. This trend varies from using low concentrations of the present unsatisfactory dyes, applying new safer dyes alternatives like brilliant blue G and infracyanine green, protecting the fovea from toxic dyes during macular hole repair surgery by applying protective viscoelastics to even exploring the ability of an intraoperative handheld OCT to evaluate the macula before and after conducting the peeling. ${ }^{24-30}$ Our study may add a new possible approach by helping the surgeon to obtain information about the borders, morphology and shape of the iERM before surgery by indicating the exact location and traction dynamics seen by OCT mapping.

The strict exclusion criteria to obtain accurate measurements from high quality photos present limitations in our study owing to the small sample size. We excluded images with patchy staining because it was difficult to outline the iERM edges. In these cases, we did not find a clearly delineated central unstained area surrounded by stained retina, but rather islands of unstained retina which could not have been reproducibly measured. This would definitely have added examiner bias to our study. In addition, the potential investigators' bias in evaluation of the morphological perspective and the non-blinded form of our study have to be considered. Nevertheless, an unprecedented clinical significance can be deduced. The identification of the presumed shape and size of the iERM may allow a safe and easy peeling process without use of dyes.

In summary, colour coded mapping by SD-OCT was used preoperatively to precisely identify the shape and configuration of the iERMs. Comparison with intraoperative ICG staining demonstrated a significant correlation with an increase of retinal thickness in the range of $440-480 \mu \mathrm{m}$ as indicated by the red coding. Our study suggests that retinal thickness mapping may guide the surgical procedure and allow the avoidance of the risks of intraoperative dyes. Our findings also highlight the substantial tractive forces of adhesive membranes over large areas of the central retina.

Acknowledgements The authors would like to thank Alessio Montuoro for technical support in computer programming

Contributors All authors have participated directly in planning and execution of the work and have approved the final version of the manuscript. BH: acquisition of data drafting and writing the article; MG: conception and design, acquisition of data, final approval; RS: analysis and interpretation of data, drafting the article; WG: acquisition of data, statistical analysis; and US-E: conception and design, revising the article critically for important intellectual content.

\section{Competing interests None.}

Provenance and peer review Not commissioned; externally peer reviewed.

\section{REFERENCES}

1. Huang D, Swanson EA, Lin CP, et al. Optical coherence tomography. Science 1991:254:1178-81.

2. Srinivasan VJ, Wojtkowski M, Witkin AJ, et al. High-definition and 3-dimensional imaging of macular pathologies with high-speed ultrahigh-resolution optical coherence tomography. Ophthalmology 2006;113:2054.e1-14.

3. Do DV, Cho M, Nguyen QD, et al. Impact of optical coherence tomography on surgical decision making for epiretinal membranes and vitreomacular traction. Retina 2007:27:552-6.

4. Schmidt-Erfurth U, Leitgeb RA, Michels S, et al. Three-dimensional ultrahighresolution optical coherence tomography of macular diseases. Invest Ophthalmol Vis Sci 2005:46:3393-402.

5. Gloesmann M, Hermann B, Schubert C, et al. Histologic correlation of pig retina radial stratification with ultrahigh-resolution optical coherence tomography. Invest Ophthalmol Vis Sci 2003;44:1696-703.

6. Georgopoulos M, Geitzenauer W, Ahlers C, et al. High-resolution optical coherence tomography to evaluate vitreomacular traction before and after membrane peeling (In German). Ophthalmologe 2008;105:753-60.

7. Glittenberg C, Krebs I, Falkner-Radler C, et al. Advantages of using a ray-traced, three-dimensional rendering system for spectral domain Cirrus HD-OCT to visualize subtle structures of the vitreoretinal interface. Ophthalmic Surg Lasers Imaging 2009; 40:127-34

8. Hassenstein A, Scholz F, Richard G. OCT in epiretinal gliosis (In German). Ophthalmologe 2005;102:127-32.

9. Falkner-Radler Cl, Glittenberg C, Binder S. Spectral domain high-definition optical coherence tomography in patients undergoing epiretinal membrane surgery. Ophthalmic Surg Lasers Imaging 2009;40:270-6.

10. Azzolini C, Patelli F, Codenotti M, et al. Optical coherence tomography in idiopathic epiretinal macular membrane surgery. Eur J Ophthalmol 1999;9:206-11.

11. Hattenbach L0, Hohn F, Fulle G, et al. Preoperative assessment of topographic features in macular pucker using high-definition optical coherence tomography (In German). Klin Monbl Augenheilkd 2009;226:649-53.

12. Suh MH, Seo JM, Park KH, et al. Associations between macular findings by optical coherence tomography and visual outcomes after epiretinal membrane removal. Am J Ophthalmol 2009;147:473-80 e473.

13. Wilkins JR, Puliafito CA, Hee MR, et al. Characterization of epiretinal membranes using optical coherence tomography. Ophthalmology 1996;103:2142-51.

14. Gomes NL, Corcostegui I, Fine HF, et al. Subfoveal pigment changes in patients with longstanding epiretinal membranes. Am J Ophthalmol 2009;147:865-8.

15. Foster RE, Petersen MR, Da Mata AP, et al. Negative indocyanine green staining of epiretinal membranes. Retina 2002;22:106-8.

16. Li K, Wong D, Hiscott $P$, et al. Trypan blue staining of internal limiting membrane and epiretinal membrane during vitrectomy: visual results and histopathological findings. Br J Ophthalmol 2003;87:216-19.

17. Gandorfer A, Messmer EM, Ulbig MW, et al. Indocyanine green selectively stains the internal limiting membrane. Am J Ophthalmol 2001;131:387-8.

18. Mori K, Gehlbach PL, Sano A, et al. Comparison of epiretinal membranes of differing pathogenesis using optical coherence tomography. Retina 2004;24:57-62.

19. Legarreta JE, Gregori G, Knighton RW, et al. Three-dimensional spectral-domain optical coherence tomography images of the retina in the presence of epiretinal membranes. Am J Ophthalmol 2008;145:1023-30.

20. Husson-Danan A, Glacet-Bernard A, Soubrane G, et al. Clinical evaluation of the use of indocyanine green for peeling the internal limiting membrane in macular hole surgery. Graefes Arch Clin Exp Ophthalmol 2006;244:291-7.

21. Ferencz M, Somfai GM, Farkas A, et al. Functional assessment of the possible toxicity of indocyanine green dye in macular hole surgery. Am J Ophthalmol 2006;142:765-70.

22. Stanescu-Segall D, Jackson TL. Vital staining with indocyanine green: a review of the clinical and experimental studies relating to safety. Eye (Lond) 2009;23:504-18. 
23. von Jagow B, Hoing $A$, Gandorfer $A$, et al. Functional outcome of indocyanine green-assisted macular surgery: 7-year follow-up. Retina 2009;29:1249-56.

24. Gale JS, Proulx AA, Gonder JR, et al. Comparison of the in vitro toxicity of indocyanine green to that of trypan blue in human retinal pigment epithelium cell cultures. Am J Ophthalmol 2004;138:64-9.

25. Shimada H, Nakashizuka H, Hattori $T$, et al. Double staining with brilliant blue $G$ and double peeling for epiretinal membranes. Ophthalmology 2009;116: 1370-6.

26. Rodrigues EB, Penha FM, de Paula Fiod Costa E, et al. Ability of new vital dyes to stain intraocular membranes and tissues in ocular surgery. Am J Ophthalmol 2010;149:265-77.
27. Feldman A, Zerbib J, Glacet-Bernard A, et al. Clinical evaluation of the use of infracyanine green staining for internal limiting membrane peeling in epimacular membrane surgery. Eur J Ophthalmol 2008;18:972-9.

28. Rizzo S, Belting C, Genovesi-Ebert F, et al. Modified technique for safer indocyaninegreen-assisted peeling of the internal limiting membrane during vitrectomy for macular hole repair. Graefes Arch Clin Exp Ophthalmol 2006:244:1615-19.

29. Lai CC, Chuang LH, Wang NK, et al. Trypan blue selective staining using whole blood for internal limiting membrane peeling during macular hole corrective surgery. Cutan Ocul Toxicol 2009;28:114-18.

30. Dayani PN, Maldonado R, Farsiu S, et al. Intraoperative use of handheld spectral domain optical coherence tomography imaging in macular surgery. Retina 2009:29:1457-68. 